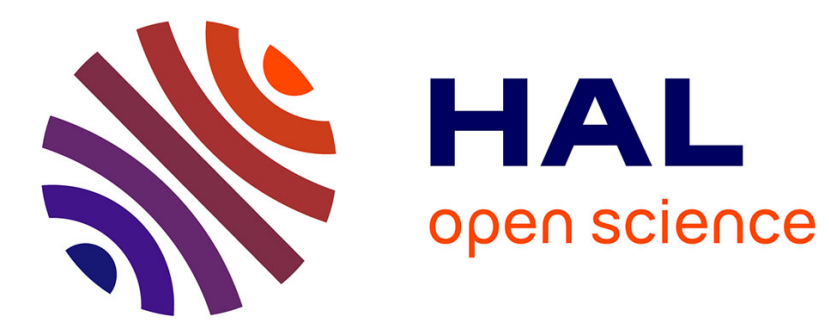

\title{
Synthesis and properties of porous ester-silica nanoparticles
}

Hung Nguyen, Alain Ibanez, Mathieu Salaun, Stéphanie Kodjikian, Philippe Trens, X. Cattoen

\section{- To cite this version:}

Hung Nguyen, Alain Ibanez, Mathieu Salaun, Stéphanie Kodjikian, Philippe Trens, et al.. Synthesis and properties of porous ester-silica nanoparticles. Microporous and Mesoporous Materials, 2021, 317, pp.110991. 10.1016/j.micromeso.2021.110991 . hal-03162361

\section{HAL Id: hal-03162361 https://hal.science/hal-03162361}

Submitted on 8 Mar 2021

HAL is a multi-disciplinary open access archive for the deposit and dissemination of scientific research documents, whether they are published or not. The documents may come from teaching and research institutions in France or abroad, or from public or private research centers.
L'archive ouverte pluridisciplinaire HAL, est destinée au dépôt et à la diffusion de documents scientifiques de niveau recherche, publiés ou non, émanant des établissements d'enseignement et de recherche français ou étrangers, des laboratoires publics ou privés. 


\title{
Synthesis and properties of porous ester-silica nanoparticles
}

\author{
Van Hung Nguyen, Alain Ibanez, ${ }^{a}$ Mathieu Salaün, Stéphanie Kodjikian, a Philippe Trens ${ }^{b}$ and Xavier Cattoëna,* \\ ${ }^{a}$ Univ. Grenoble Alpes, CNRS, Grenoble INP, Institut Néel, 38000 Grenoble, France. \\ ${ }^{b}$ ICGM University of Montpellier, CNRS, ENSCM, Montpellier, France \\ Xavier.cattoen@,neel.cnrs.fr
}

\begin{abstract}
:
Ester-silica nanoparticles (NPs) were prepared using a facile two-surfactant synthesis route in neutral medium involving silica (tetraethyl orthosilicate, TEOS) and organosilica (3-(trimethoxysilyl)propyl 5(trimethoxysilyl)pentanoate, $\mathbf{P}_{\mathrm{Me}}$ ) precursors. The ester hydrolysis generates carboxylic acid groups, which confer a strongly negative $\zeta$ potential to the NPs at neutral $\mathrm{pH}$. The extent of hydrolysis during the synthesis was quantified by FTIR. The NP size was shown to be highly dependent on hydrodynamic factors such as stir bar size and stirring speed, enabling to fine-tune the NP diameter. The textural properties of the NPs could be monitored using appropriate fraction $x$ of silica precursor, $x=[$ TEOS $] /\left([\mathrm{TEOS}]+2\left[\mathrm{P}_{\mathrm{Me}}\right]\right)$. For NPs with a silica fraction $x$ higher than 0.5 , a significant porosity could be observed, with mesoporosity appearing for $x \geq 0.7$. The physicochemical and textural properties of the prepared materials open applications for water pollution abatement of specific organic compounds. This is exemplified by the efficient adsorption of rhodamine B from the liquid phase by the prepared NPs due to their physicochemical and textural parameters, including a high specific surface area and porosity, strong electrostatic forces and lipophilicity of the pore walls.
\end{abstract}

\section{Introduction:}

Organic-inorganic hybrid silicas, or organosilicas, are being widely investigated for applications in catalysis,[1] optics, [2,3] pharmaceutics [4] or depollution. [5] In fact, they advantageously combine the rigidity and tunability of the inorganic matrix with the tunable functionality of the organic components. Many types of organosilicas exist, depending on the existence of covalent linkage between both components and on the synthesis mode. For covalent organosilicas, one can distinguish bridged silsesquioxanes[6] and their mesoporous analogs called periodic mesoporous organosilicas[7,8] (PMOs), obtained solely from the hydrolysis and condensation of organosilanes, without the addition of silica precursors, and which inherently feature the maximal loading in organic functionalities. Other organosilicas are obtained through the sol-gel process from mixtures of silica and silsesquioxane precursors, with amounts of organic groups usually lower than $10 \mathrm{~mol} \%$ with respect to silicon atoms. Finally, many authors use grafting procedures to anchor covalently organic groups on the surface of preformed silica materials. These distinct procedures therefore lead to different types of materials, with organic functionalities either pending from the surface or incorporated within the matrix.[9-11] The synthesis of organosilica materials at the nanoscale is much less documented as the control of the morphology and size of nanoparticles (NPs) is not straightforward.[12] Upon decreasing the size, the surface to volume ratio becomes higher, which is interesting for applications in depollution or catalysis, as issues related to diffusion through the material are lowered.[13] Furthermore, having particles with a size lower than $200 \mathrm{~nm}$ allows them to circulate in blood for a significant duration, 
which enables applications in nanomedicine for targeted drug delivery or bioimaging. [14] For all these applications, the organosilica NPs are most often obtained by grafting organosilanes on preformed silica NPs, the formation of bridged silsesquioxanes[15] or PMO NPs[16] being far less documented. Indeed, the controlled formation of NPs is disturbed by the presence of high loading of organics. This becomes even more challenging for the synthesis of PMO NPs, [16] for which mostly simple, rigid organic groups have been used in the scaffold. The recent preparation of porphyrin-based[17] or cationic PMO NPs with flexible organic linkers[18] are therefore very interesting examples. In the latter case, a two-surfactant method was employed to achieve the nanoscale and the formation of regular pores, using a mixture of neutral and ionic surfactants as structure-directing agents. This technique was earlier introduced for the preparation of phenylene-based PMOs, with neutral and cationic surfactants.[19] Introducing organics within the walls of NPs is indeed an interesting way to anchor the maximal loading in functionalities, $[17,18]$ or to modify the hydrophilic-hydrophobic balance of the pores in order to increase the drug loading capacity for instance,[20] or to prepare breakable NPs.[20-25] In particular, bridged silsesquioxane,[26] PMO and organosilica NPs were made degradable thanks to high amounts of cleavable groups within the organic sub-network. These groups include disulfide and tetrasulfide, imine, amides and esters. The latter were used for degradable thin organosilica shells around liposomes thanks to enzymatic cleavage.[27] Indeed, ester functionalities are particularly interesting in biomedicine as esterases are overexpressed in certain cancer cell lines.[28] Thus, several groups described nanosystems using the cleavage of ester functions to trigger a particular action: stoppers for drug delivery,[29] fragmentation of a thin organosilica shell around a liposome[27] or charge reversal upon ester cleavage.[30] In the few papers dealing with mesoporous silica nanoparticles (MSN), the ester groups have been introduced by grafting around a pre-formed MSN. However, for a use as a structural unit that may be broken by hydrolysis after use,[20,31] it is important to have a high amount of cleavable functions and to incorporate the ester groups throughout the whole volume of particles. Therefore, the ester-containing precursors have to be incorporated by co-condensation with a silicate or silsesquioxane precursor. However, the co-condensation strategy usually suffers several drawbacks such as the limited amount of organic groups that can be incorporated while preserving a significant mesoporosity, and additionally in the present case, the high pH used for most MSN synthesis. [32] Indeed, it is noteworthy that esters are particularly sensitive functional groups that readily hydrolyze under acidic or basic $\mathrm{pH}$. In this study, we therefore chose to adapt a synthesis of MSNs in an aqueous buffer at $\mathrm{pH} 7.0$ using a mixture of two surfactants, Brij C10 and CTAB[33] that has proven suitable for the incorporation of high amounts of organic moieties in mesoporous organosilica NPs.[13] The NP preparation with tunable diameter incorporating high amount of ester groups is described by varying several experimental factors and the molar fractions of silicate (TEOS, tetraethyl orthosilicate) and silsesquioxane ( $\mathbf{P}_{\mathrm{Me}}$, see formula in Figure 1) precursors specified by $x=\frac{[T E O S]}{[T E O S]+2[P M e]}$. We also investigated the extent of partial hydrolysis of the ester functions by Fourier transform infrared spectroscopy (FTIR). We discovered that the washing steps induced a significant amount of ester cleavage, leading to porous NPs with significant loading of carboxylic acid functions, useful for the entrapment of cationic salts or organic compounds.[34]

\section{Experimental section:}

Materials: The precursor $\mathbf{P}_{\mathrm{Me}}$ (3-(trimethoxysilyl)propyl 5-(trimethoxysilyl)pentanoate, see formula in Figure 1) was synthesized according to a reported procedure.[27] Hexadecyltrimethylammonium bromide (CTAB, A15235) was purchased from Alfa Aesar. Brij C10 and all other reagents were purchased from Sigma Aldrich. Gradient HPLC grade water was used for all syntheses and washings. The centrifugations were performed using a Beckman Allegra 64R apparatus in $50 \mathrm{~mL}$ tubes, or using an Eppendorf 5430 equipment at $17500 \mathrm{rpm}$ for $1.5 \mathrm{~mL}$ microtubes.

Methods: FESEM images were recorded using a Zeiss Ultra+ scanning electron microscope. $3 \mu \mathrm{L}$ of a dilute NP suspension were dried on a doped silicon wafer for observation. A voltage of $3 \mathrm{kV}$ was used at a working distance of $3 \mathrm{~mm}$. Transmission electron microscopy (TEM) was performed on a Philips CM300 microscope operating at $300 \mathrm{kV}$. The TEM grid was prepared by depositing a dilute drop of NP suspension in ethanol on a carbon-coated copper grid. Images were collected on a TemCam F416 TVIPS, which is a 
rapid, large field of view, high-resolution and high dynamic range CMOS camera (4k $\times 4 \mathrm{k}, 16$ bits). Nitrogen sorption experiments were performed at $77 \mathrm{~K}$ using a Micromeritics Tristar 3000, after degassing samples $12 \mathrm{~h}$ at $80^{\circ} \mathrm{C}$ under secondary vacuum $\left(10^{-5}\right.$ Torr). The specific surface areas were calculated using the BET model in the range $0.15<p / p^{\circ}<0.35$, taking $0.162 \mathrm{~nm}^{2}$ as cross-sectional area for the adsorbed nitrogen molecules. The t-plot method was used to determine the micropores volumes, taking the Harkins and Jura equation as reference sorption curve. The mesopores volumes were further determined by subtracting the obtained micropores volume to the full pores volume considered at $\mathrm{p} / \mathrm{p}^{\circ}=0.6$. Thermogravimetric analyses (TGA) and differential thermal analyses (DTA) were carried out on a SETARAM TAG 16 instrument, using $3 \mathrm{mg}$ samples within an alumina crucible under an argon-oxygen (4:1) atmosphere with a heating rate of 10 ${ }^{\circ} \mathrm{C} \mathrm{min}^{-1}$. These TGA and DTA characterizations were coupled to mass spectrometry of gas exhausts using an HIDEN analytical apparatus (QGA-HAL201-RC). The zeta potentials $(\zeta)$ were determined using a Wallis analyzer (Cordouan, France) in $2 \mathrm{mM}$ acetate of phosphate buffers, after checking that the size measured by dynamic light scattering was less than $200 \mathrm{~nm}$ (DLS, cumulant fit in intensity measured on a Cordouan Nanokin apparatus). Absorption spectra were recorded using a SAFAS Xenius XC Cuvette spectrofluorometer. FTIR spectra were measured using a Thermo Fisher Nicolet ${ }^{\mathrm{TM}}{ }^{\mathrm{N}} \mathrm{N}^{\mathrm{TM}} 10$ spectrometer in transmission mode on $\mathrm{KBr}$ pellets obtained from $0.5 \mathrm{mg}$ of NPs pressed within $140 \mathrm{mg}$ of dried potassium bromide.

Typical synthesis of ester-silica NPs with $x=0.5$ : In a $100 \mathrm{~mL}$ round bottom flask equipped with a $12 \times 25 \mathrm{~mm}$ oval-shaped stir bar were added Brij C10 (150 mg, $0.22 \mathrm{mmol})$, CTAB (230 mg, $0.63 \mathrm{mmol})$, potassium dihydrogen phosphate $(214 \mathrm{mg}, 1.57 \mathrm{mmol})$ water $(67 \mathrm{~mL})$ and a sodium hydroxide solution $(2.0 \mathrm{M}, 0.45$ $\mathrm{mL}, 0.90 \mathrm{mmol}$. The mixture was heated to $95{ }^{\circ} \mathrm{C}$ at a stirring rate of $500 \mathrm{rpm}$ until all components had dissolved. Then, a mixture of $\mathbf{P}_{\mathrm{Me}}(0.50 \mathrm{~mL}, 1.3 \mathrm{mmol})$ and TEOS $(0.50 \mathrm{~mL}, 2.4 \mathrm{mmol})$ was added and the resulting solution was kept for $8 \mathrm{~h}$ at $95{ }^{\circ} \mathrm{C}$ under $500 \mathrm{rpm}$ stirring. After cooling, the suspension was centrifuged at $41000 \mathrm{~g}$ for 30 minutes, and the supernatant was discarded. $25 \mathrm{~mL}$ of an ethanolic solution of ammonium nitrate $(6 \mathrm{~g} / \mathrm{L})$ was added to each tube. After sonication $\left(30 \mathrm{~min}\right.$ at $\left.50{ }^{\circ} \mathrm{C}\right)$, the tubes were centrifuged $(30 \mathrm{~min}$ at $41000 \mathrm{~g}$ ). This operation was repeated twice, then the NPs were washed similarly with water, ethanol then acetone, and finally dried at $60{ }^{\circ} \mathrm{C}$ overnight.

Loading of ester-silica nanoparticles: $10 \mathrm{mg}$ of dried NPs were suspended in $5.0 \mathrm{~mL}$ of deionized water by sonication. In three Eppendorf tubes were placed $500 \mu \mathrm{L}$ of this suspension (1.0 mg of NPs), and $500 \mu \mathrm{L}$ of a rhodamine B solution $\left(2.0 \mathrm{mg} \mathrm{mL}^{-1}, 1.0 \mathrm{mg}\right)$. After mixing with a vortex mixer, the tubes were kept at $25{ }^{\circ} \mathrm{C}$ for $24 \mathrm{~h}$, then centrifuged. The supernatants were placed in a $25 \mathrm{~mL}$ volumetric flask, then the NPs were washed with water 3-4 times until the supernatants were clear. All supernatants were combined in the volumetric flask, and the volume was adjusted to $25 \mathrm{~mL}$. The amount of rhodamine B encapsulated was determined by absorbance at $554 \mathrm{~nm}$ by comparison with the initial rhodamine B solution. The Loading Capacity $(\mathrm{wt} \%)$ is expressed as: [mass of loaded dye/(mass of loaded dye + mass of NPs)] $\times 100$. These experiments were performed in triplicate. 

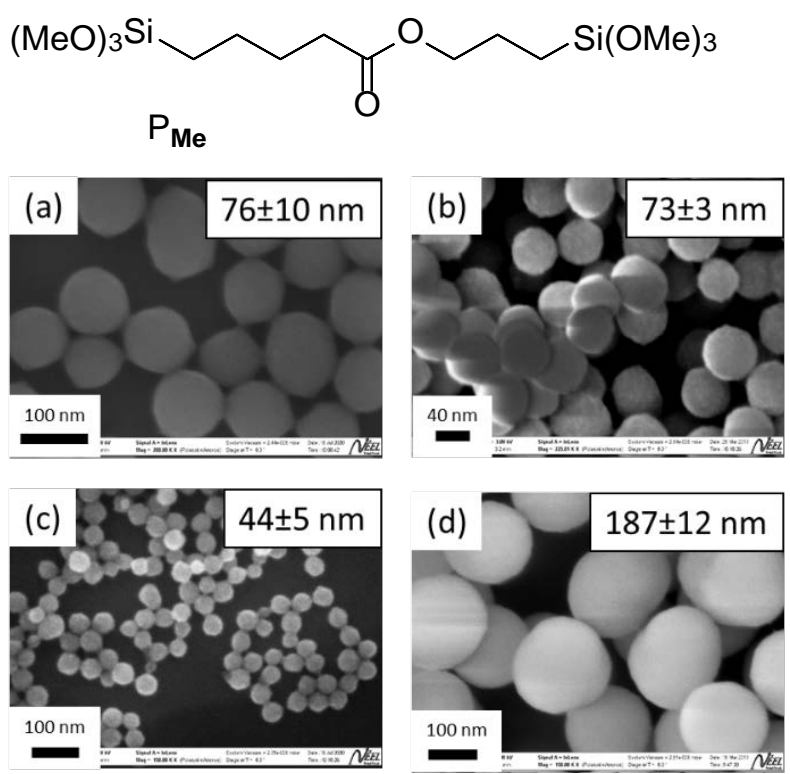

Figure 1: Formula of the precursor $\mathbf{P}_{\mathrm{Me}}$, and SEM images of NPs obtained with $\mathrm{x}=0$ (a); $\mathrm{x}=0.5$ (b); $\mathrm{x}=$ 0.7 (c) and $x=1(d)$.

\section{Results and discussion:}

In order to include the maximal amount of ester-silica in NPs, while preserving the integrity of the ester functions, we adapted a synthesis in neutral conditions developed for MSNs[33]. This has recently allowed us to include functional porous organosilica NPs at high loading.[13] TEOS and $\mathbf{P}_{\text {Me precursor }}$ were co-condensed in an aqueous phosphate buffer at $\mathrm{pH} 7.0$ in the presence of a surfactant mixture composed of CTAB and Brij $\mathrm{C} 10$ at $95^{\circ} \mathrm{C}$. The molar ratios were the following: Brij $\mathrm{C} 10$ : CTAB : TEOS : $\mathbf{P}_{\text {Me }}: \mathrm{H}_{2} \mathrm{O}=1: 2.9: 22.7 \times x: 11.4 \times(1-x): 16900$, with $x$ varied from 0 to 1 , so that the overall concentration in silicon atoms was kept constant. Whatever the molar fraction of precursors $x$, monodisperse NPs with sizes between 40 and $190 \mathrm{~nm}$ were obtained (Figure 1), which illustrates the potentiality of the two surfactants methods to prepare ester-silica NPs.[13,18,19,33] In all cases, the morphology of the obtained NPs was mostly spherical, with a rough surface. We found out that the NP size was highly dependent on the hydrodynamic factors such as size of the flask, stirring speed and size of the stir bar. Indeed, when varying only the stirring speed all other parameters being constant, the size of NPs with $x=0.5$ can be tuned from 40 to $115 \mathrm{~nm}$ (Figure 2a-c), the biggest NPs being obtained at slower speed. Similarly, when the stir bar size is decreased, bigger NPs can be obtained. It is noteworthy that similar results are obtained for pure silica samples (Figure S1). This dependency on hydrodynamic factors is an indication of the control of the NP formation by the nucleation step. Stronger hydrodynamic forces lead to higher nucleation frequency, thus higher number of nuclei are produced limiting the growth of each NP. Such an influence of stirring is commonly encountered in crystallization processes.[35] 

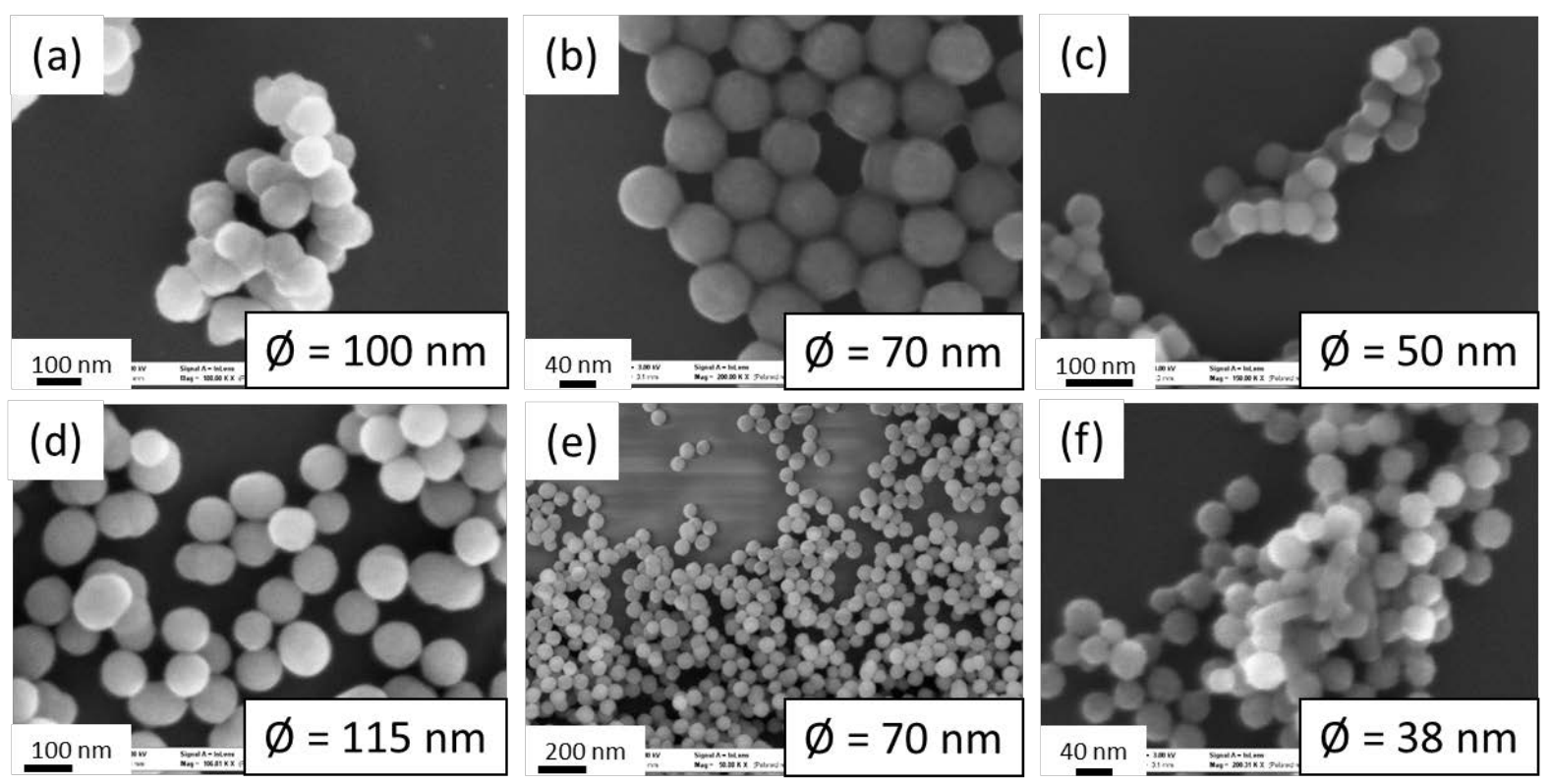

Figure 2: SEM micrographs of ester-silica NPs and associated average diameters obtained with different magnetic stir bars or at different stirring speed for $x=0.5$, the other synthesis parameters being constant. For stir bar dimensions of $12 \times 25 \mathrm{~mm}$, stirring speed of $200 \mathrm{rpm}$ (a); $500 \mathrm{rpm}$ (b) and $1000 \mathrm{rpm}$ (c). For a stirring speed of $500 \mathrm{rpm}$, stir bar dimensions: of $5 \times 15 \mathrm{~mm}$ (d); $12 \times 25 \mathrm{~mm}$ (e) and $20 \times 40 \mathrm{~mm}$ (f).
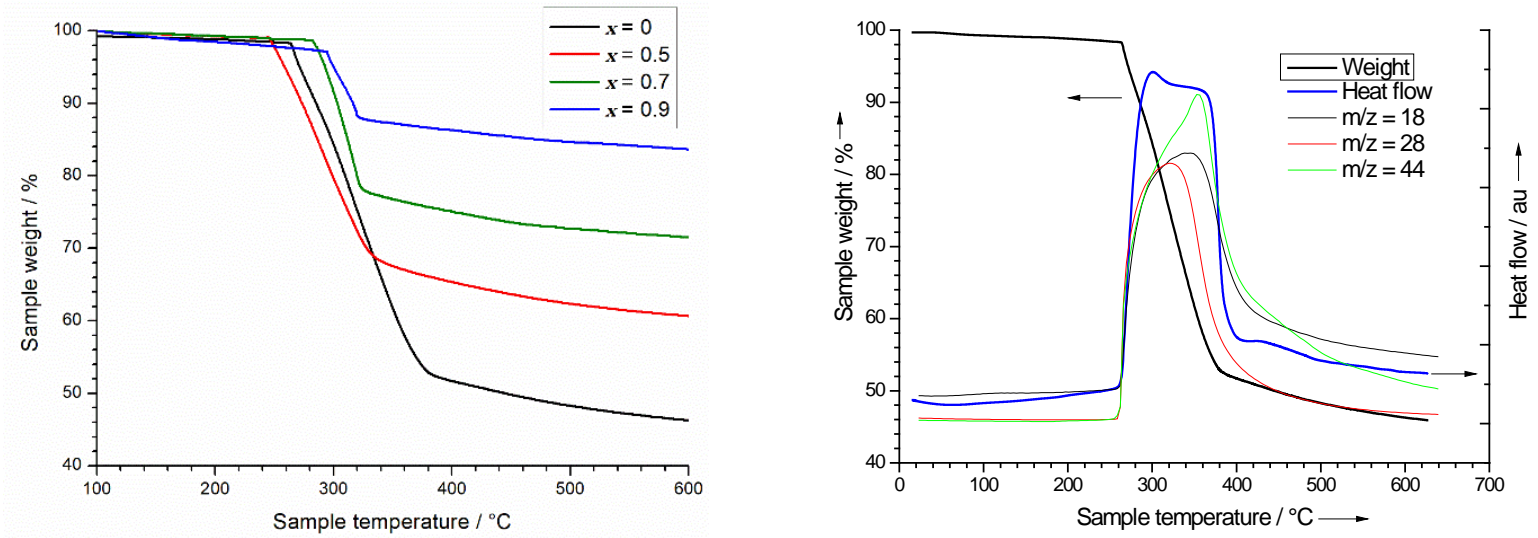

Figure 3: (Left) Compared thermogravimetric analyses in air $\left(10^{\circ} \mathrm{C} / \mathrm{min}\right)$ of samples with $x=0$ (black), $x$ $=0.5$ (red), $x=0.7$ (green) and $x=0.9$ (blue); (Right) Coupled analyses of TGA (black line), DTA (blue curve, exothermic peak) and mass spectrometry of gas exhausts during thermal analyses of sample $x=0$, water $(\mathrm{m} / \mathrm{z}=18$, black thin line), carbon monoxide $(\mathrm{m} / \mathrm{z}=28$, red) and carbon dioxide $(\mathrm{m} / \mathrm{z}=44$, green).

\begin{tabular}{llll}
\hline$x$ & Calculated weight loss $(\%)$ & Weight loss $(\%)$ at $600{ }^{\circ} \mathrm{C}$ & Onset $\left({ }^{\circ} \mathrm{C}\right)$ \\
\hline 0 & 51.2 & 52.1 & 260 \\
0.5 & 41.1 & 38.2 & 245 \\
0.7 & 32.6 & 29.4 & 280 \\
0.9 & 16.0 & 14.3 & 295 \\
\hline
\end{tabular}

Table 1: Data extracted from the thermogravimetric analyses $\left(10^{\circ} \mathrm{C} / \mathrm{min}\right)$.

The TGA analysis, (Figure 3, left) for samples with different $x$ values, shows an initial loss of adsorbed solvents and water until $240^{\circ} \mathrm{C}$. Then an abrupt weight loss is observed from $245-295{ }^{\circ} \mathrm{C}$ until ca $400{ }^{\circ} \mathrm{C}$ corresponding to the combustion of the organics within the framework, as indicated by the $\mathrm{CO}, \mathrm{CO}_{2}$ gas exhausts analyzed by mass spectrometry (Figure 3, right). Finally, water is slowly released until $600{ }^{\circ} \mathrm{C}$, which 
corresponds to further condensation in the framework with release of water. For the three samples analyzed, the weight loss that is found experimentally at $600{ }^{\circ} \mathrm{C}$ agrees well with that calculated based on a full condensation at silicon (Table 1). This is an evidence of the full incorporation of both silica and silsesquioxanes inside the framework. It is noteworthy that a single event takes place from $245-295^{\circ} \mathrm{C}$, with no initial decarboxylation as no carbon dioxide is evolved before water, as shown by TGA-MS, Figure 3, right. The TGA and DTA were also recorded for the samples before surfactant extraction (Figure S2): for $x=0.5, x=0.7$ and $x=0.9$, the difference in weight loss before and after surfactant removal increased with $x$, with values of $8.2 \%, 9.6 \%$ and $12.2 \%$, respectively. This evolution agrees with an increase in pore volume, (Table 2), however with values that are not proportional, indicating that after washing the NPs simply twice with ethanol, the surfactants were not only located inside the pores, but also at the NP surface. The onsets of combustion drastically change depending on $x$ : for $x=0.5$ and 0.7 the onset is lower for the extracted sample, while for $x=0.9$ an opposite behavior is observed.

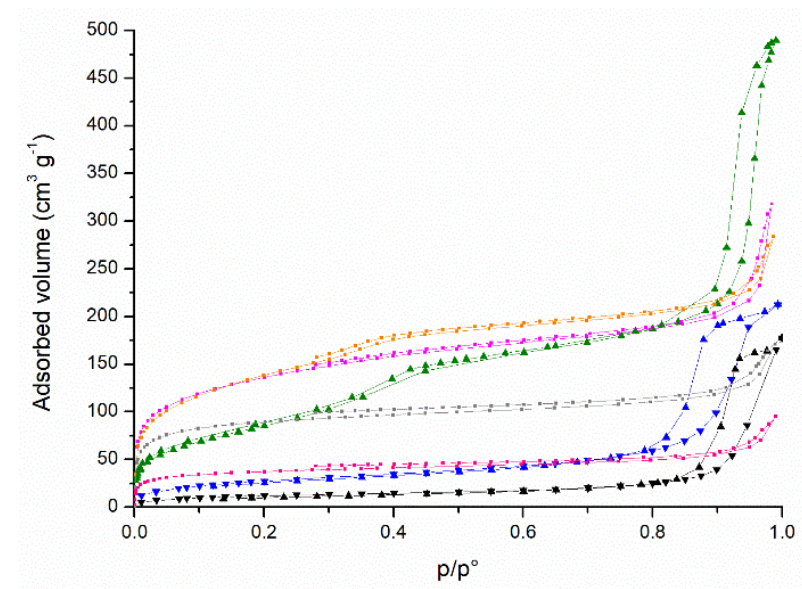

Figure 4: $\mathrm{N}_{2}$-sorption isotherms of NPs obtained with $x=0$ (black); $x=0.5$ (blue); $x=0.6$ (red); $x=0.7$ (grey); $x=0.8$ (pink); $x=0.9$ (orange) and $x=1$ (green).

The textural properties of the different NPs were probed by $\mathrm{N}_{2}$-sorption experiments (Figure 4). Depending on the organic content of the material investigated, different sorption isotherms shapes can be observed. In the case of the pure ester-silica NPs $(x=0)$, a type II sorption isotherm can be seen, along with a hysteresis loop at high relative pressure. It can be concluded that this material is made of non-porous NPs. The corresponding specific surface area, determined using the BET transform of the sorption isotherm is $45 \mathrm{~m}^{2}$ $\mathrm{g}^{-1}$, thus attributable to the external surface of the ester-silica spheres (Table 2). The hysteresis loop observed at high relative pressure (black curve, Figure 4) belongs to the H2 type according to the IUPAC classification, which usually describes the aggregation of particles. It can be deduced that this material is made of aggregated non-porous NPs.

When $x$ increases, the shape of the sorption isotherms (Figure 4) change, thus implying changes in the textural properties of the obtained NPs. For values of $x$ located between 0.5 and 0.7 , the greater extent of the uptake at very low relative pressure, along with a flat plateau at intermediate relative pressure, is reminiscent of the type I sorption isotherms, typical for microporous materials. The hysteresis loops observed at high relative pressure are different, depending on the value of $x$. In the case of $x=0.5$, a H2 type is obtained, which indicates the occurrence of aggregated particles. In the case of $x=0.6$ or $x=0.7$, the hysteresis loop is very small (H3 type), which shows that the corresponding materials can undergo a slight swelling upon adsorption. The sorption isotherm obtained for $x=0.8$ is different to those described above. The uptake at very low pressure is very important and the knee of the sorption isotherm is located at $\mathrm{p} / \mathrm{p}^{\circ}=0.15$, which strongly suggests the occurrence of small mesopores. This is consistent with the slope of the curve at intermediate pressure, which indicates that the monolayer multilayer sorption process is quantitative. In this case, the hysteresis loop is similar to that obtained for $x=0.7$. 
The curve obtained for $x=0.9$ shows a clear uptake at intermediate relative pressure $\left(\mathrm{p} / \mathrm{p}^{\circ}=0.35\right)$ and a saturation plateau at higher relative pressure. Because the sorption isotherms obtained in the case of $x=0.8$ and $x=0.9$ are very similar up to $\mathrm{p} / \mathrm{p}^{\circ}=0.3$, it can be deduced that the latter material has some residual micropores. This sorption isotherm should therefore be classified as intermediate between Types I and IV. For $x=1$, the sorption isotherm obtained is typical for mesoporous materials (Type IV), with a strong adsorbed amount at low pressure, followed by a high uptake at intermediate pressure followed by a rather flat plateau.

The corresponding textural properties are reported in Table 2. When $x$ increases, the specific surface area rises from 45 up to $492 \mathrm{~m}^{2} \mathrm{~g}^{-1}$ for $x=0$ and $x=0.9$, respectively. In the case of $x=1$, which corresponds to pure silica, the specific surface area decreases down to $332 \mathrm{~m}^{2} \mathrm{~g}^{-1}$ (Figure 5a). The curve obtained when the micropores volume is plotted versus the silica fraction, $x$, has a bell-like shape (Figure $5 \mathrm{~b}$ ). At the same time, the curve corresponding to the mesopore volume (Figure 5c) shows an increase up to $x=1$. Thus, it can be deduced that the mesoporosity is generated at the expense of the microporosity in NPs. In fact, the adsorbed amount is usually constituted by the species located on the overall surface as well as those condensed in the pores (if any) of a material. Consequently, as the micropores are transformed into mesopores for increasing $x$ ratios, the specific surface area decreases along with the micropores surface. These results therefore confirm the qualitative observations made above.

It is noteworthy that PMOs and related materials are usually prepared from simple, small organosilanes, while large amounts of high molecular weight organosilanes often strongly decrease the porosity.[7,16] Therefore, in the present case, the specific surface area increases with the proportion of TEOS in the starting mixture. Additionally, the average mesopores pore size for the materials with $x>0.7$ has been determined using the BJH model (Figure S3). The obtained value $(\sim 3 \mathrm{~nm})$ agrees with a templating from CTAB micelles, the Brij C10 surfactant being significantly longer than the CTA ${ }^{+}$cation. Thus, these materials constitute a rare example of porous organosilica NPs obtained with a high content of organic groups.

Table 2: Textural properties of the different NPs.

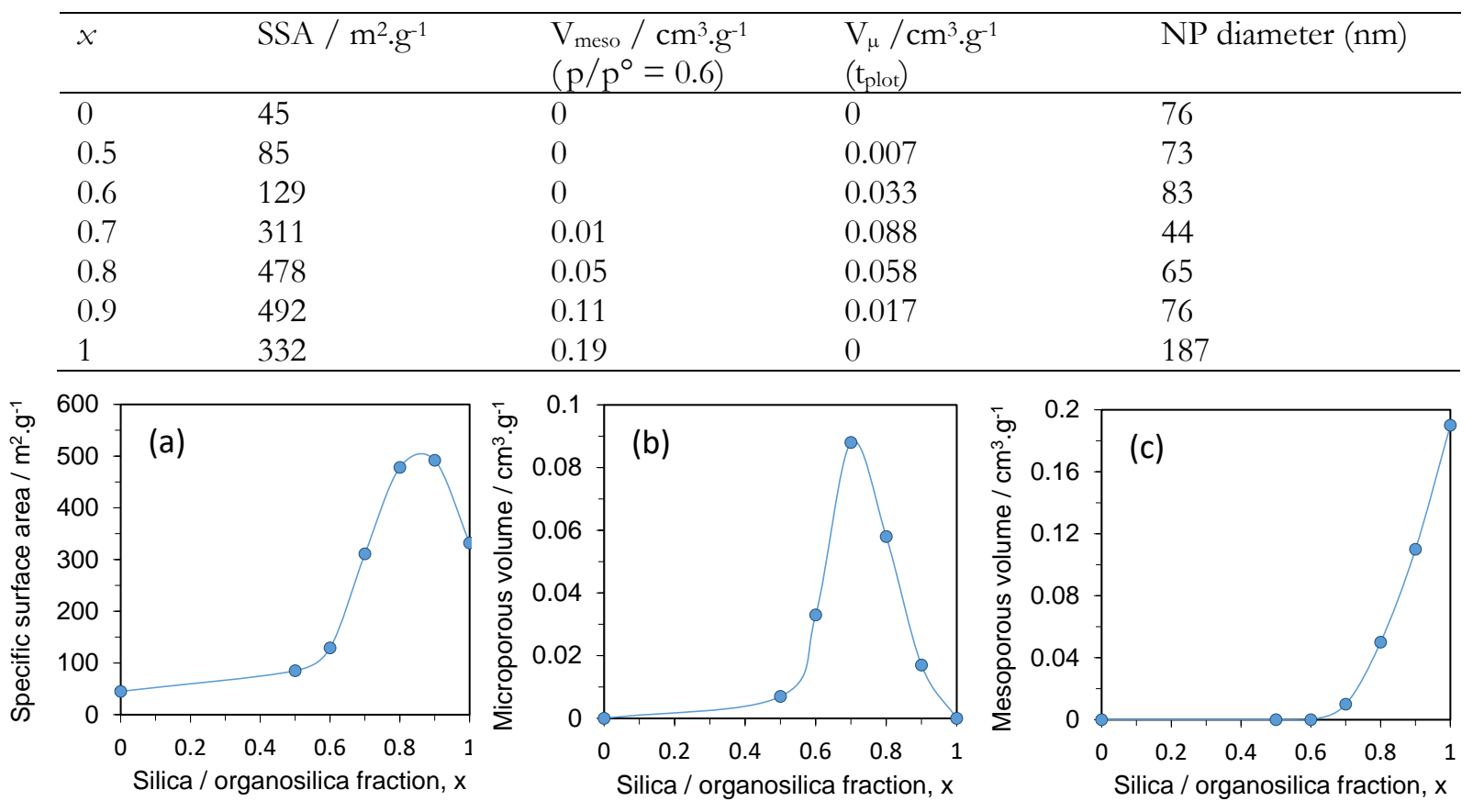

Figure 5: Textural properties of NPs versus silica fraction, $x$. Specific surface area (a); microporous (b) and mesoporous (c) volumes. 

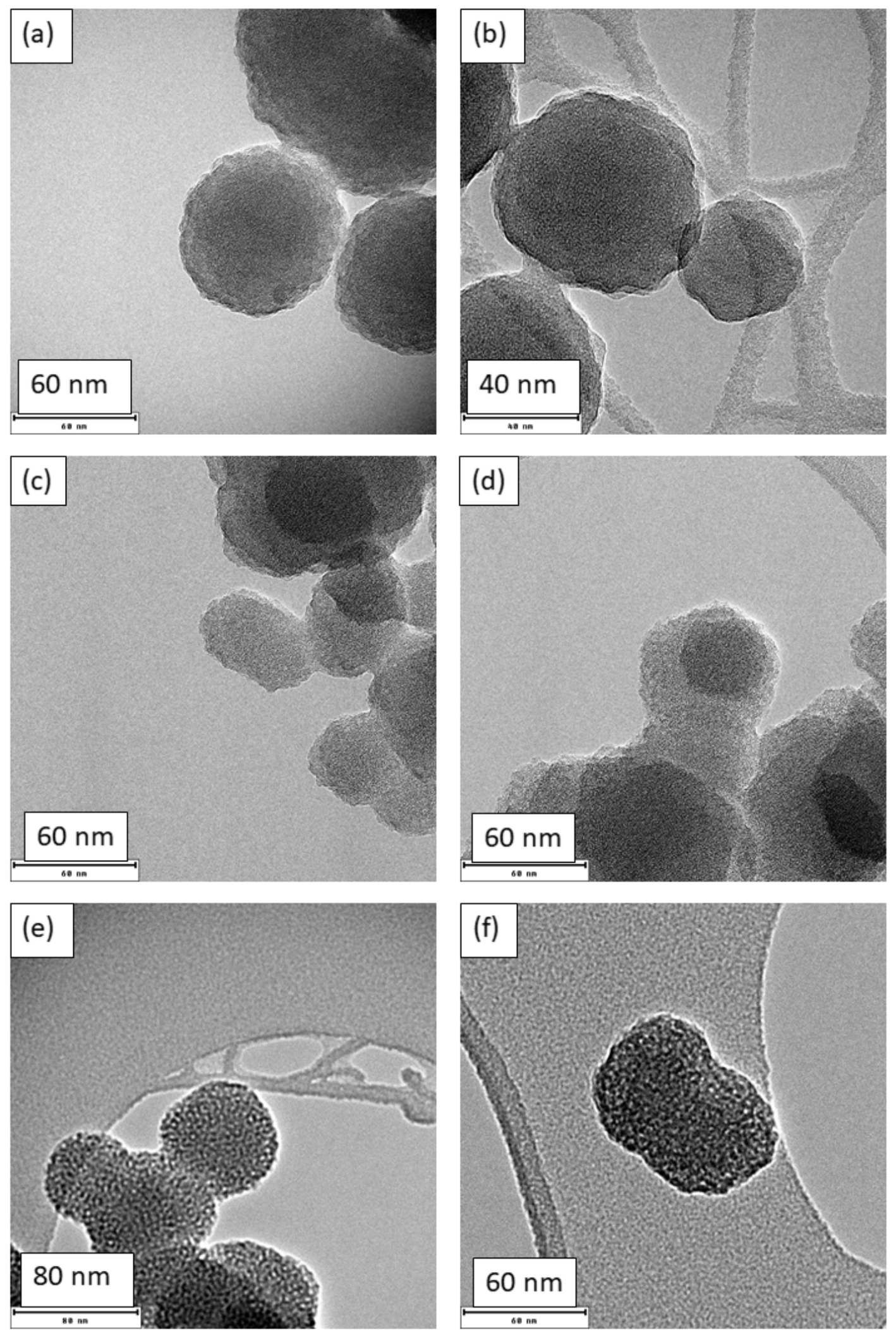

Figure 6: TEM micrographs of NPs obtained with $x=0.7(\mathrm{a}, \mathrm{b}), x=0.8(\mathrm{c}, \mathrm{d})$ and $x=0.9(\mathrm{e}, \mathrm{f})$, evidencing the transition from microporosity to mesoporosity. 
The TEM micrographs (Figures 6 and S4) confirm the above results: for $x=0$ (pure silsesquioxanes), dense NPs are observed, with unobservable porosity. However, for $x=0.5$, a slight, non-organized porosity together with surface roughness are visible (Figure S4), while for $x=0.7$ and $x=0.8$, microporosity is clearly visible (Figure $6 \mathrm{a}-\mathrm{d}$ ). This translates into larger pores for $x=0.9$ (Figure $6 \mathrm{e}-\mathrm{f}$ ), in agreement with the data obtained from $\mathrm{N}_{2}$-sorption. The lack of pore ordering is in agreement with the previous studies[13,19,33] that involved this two-surfactant synthesis for silica, while no sharp peak is visible by low-angle X-ray scattering (Figure S5). For $x \geq 0.8$, the broad peak at low angle is typical of the presence of non-organized mesopores.

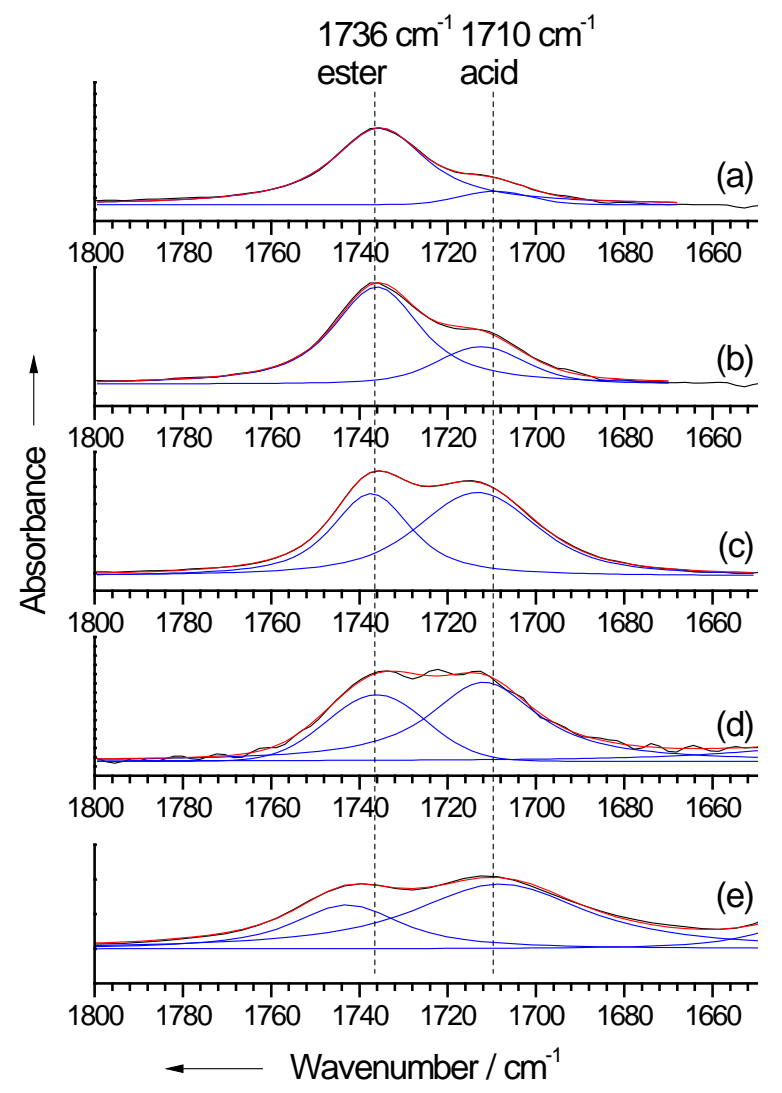

Figure 7: FTIR spectra of NPs (red curves) and corresponding deconvolutions (Voigt functions in blue) after washing for $x=0$ (a); $x=0.5$ (b); $x=0.7$ (c); $x=0.9$ (d). FTIR spectrum of a $1: 1 \mathrm{~mol} / \mathrm{mol}$ mixture of allyl pentenoate and pentenoic acid (e).

The chemical composition was further studied by FTIR (Figure S6): the broad and prominent double band at 1000-1200 $\mathrm{cm}^{-1}$ is ascribed to the symmetrical and anti-symmetrical stretching vibrations of the silicate framework, while the presence of the $\mathrm{C}=\mathrm{O}$ function is confirmed by a peak at $c a 1730 \mathrm{~cm}^{-1}$. The removal of the surfactant is clearly visible by the strong decrease of the peaks at $2800-2900 \mathrm{~cm}^{-1}$, which do not completely vanish as $\mathrm{CH}_{2}$ groups are still present in the organosilica structure. A closer look at the $\mathrm{C}=\mathrm{O}$ band between 1800 and $1650 \mathrm{~cm}^{-1}$ enables to investigate the preservation of the ester functions in the framework of the organosilica NPs (Figure 7). Indeed, the ester group presents an intense band at $1730 \mathrm{~cm}^{-}$ ${ }^{1}$ while after hydrolysis, the carboxylic acid vibrations absorb at $1710 \mathrm{~cm}^{-1}$. This can be visualized in the FTIR spectra of allyl pentenoate and pentenoic acid mixture. In the mixture of these two compounds in a 1:1 molar ratio acid (Figure 7e), it can be seen that the carboxylic acid function absorbs more intensely than the ester, by a factor 2.4. Therefore, the amount of remaining ester in the different materials can be quantified after deconvolution of the $1550-1800 \mathrm{~cm}^{-1}$ region of the spectra with Voigt functions, knowing the relative response of the ester and carboxylic acid groups. The FTIR spectra (Figure 7) show that the obtained NPs are partially hydrolyzed, from $4 \%$ to $60 \%$. The trend shows that NPs with the highest organic content (low $x$ values) present a lower hydrolysis ratio than those where the silsesquioxanes are more diluted 
within silica (high $x$ values). At first sight, this trend parallels the specific surface area of NPs, the pure silsesquioxanes being non-porous, while the ones with high $x$ values present a significant porosity. However, no obvious explanation can relate the porosity with the hydrolysis rate. To better understand the hydrolysis of the ester functions, which should not be straightforward at $\mathrm{pH} 7$, even at $95{ }^{\circ} \mathrm{C}$, we followed the FTIR spectra of NPs in the case of $x=0.5$ at different steps of the NP preparation. From the FTIR spectra (Figure 8), it appears that $c a 14 \%$ of the ester functions have been hydrolyzed after $8 \mathrm{~h}$ at $95^{\circ} \mathrm{C}$, the remaining acid being formed mainly during the first washing with ammonium nitrate, while further washings did not affect the acid/ester ratio. Finally, the reaction was carried out at $60{ }^{\circ} \mathrm{C}$ or at $35{ }^{\circ} \mathrm{C}$ for $24 \mathrm{~h}$ for $x=0.5$. Satisfactorily, the acid content just after synthesis decreased from $14 \%$ to $12 \%$ and $8 \%$ for reactions carried out at 95,60 and $35^{\circ} \mathrm{C}$, respectively, while the NP size remained constant independently of the temperature. However, during the washing steps, the ester content decreased to the same values as for the reaction carried out at the highest temperature.

\begin{tabular}{cccc}
\hline$x$ & $\begin{array}{c}\text { Final ester } \\
\text { content }(\%)\end{array}$ & $\begin{array}{c}\text { Amount of acid } \\
\text { functions } \\
(\mathrm{mmol} / \mathrm{g})\end{array}$ & $\begin{array}{c}\text { Rhodamine B } \\
\text { Loading Capacity } \\
(\mathrm{wt} \%)\end{array}$ \\
\hline 0 & 96 & 0.14 & 7.0 \\
0.2 & 94 & 0.20 & 8.0 \\
0.5 & 79 & 0.36 & 6.4 \\
0.7 & 77 & 0.87 & 10.1 \\
0.9 & 41 & 0.50 & 9.8 \\
1 & - & 0 & 4.7 \\
\hline
\end{tabular}

Table 3: Chemical composition of the NPs and loading capacity for rhodamine B.

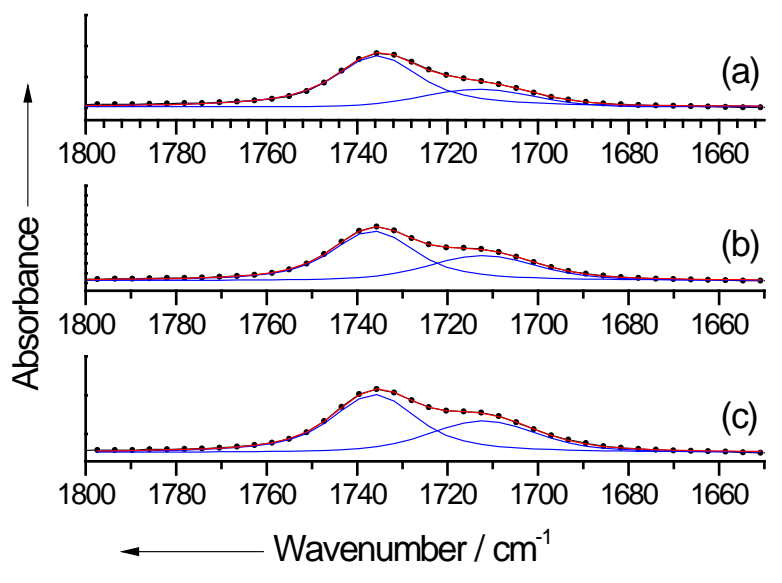

Figure 8: Evolution of FTIR spectra (red curve) and deconvolutions (Voigt functions in blue) during the washing procedure for NPs with $x=0.5$, as synthesized NPs (a); after the first washing with ammonium nitrate (b); after the third washing with ammonium nitrate (c).

Owing to the presence of the carboxylic acid functions, the NPs can be easily dispersed at $\mathrm{pH} 7.4$ for concentrations of $c a 1 \mathrm{mg} / \mathrm{mL}$ thanks to a strongly negative zeta potential, $\zeta$, the acid functions being mainly deprotonated at this $\mathrm{pH}$. As seen in related micron-sized particles,[36] the evolution of the $\zeta$ potential 
depending on the $\mathrm{pH}$ (Figure 8) shows that the ester-silica NPs are much more negative than the similar silica NPs obtained with $x=1$ (-44 to $-34 \mathrm{mV} v s-20 \mathrm{mV}$ for ester-silica and silica, respectively at $\mathrm{pH}$ 7.4). Moreover, the point of zero charge is shifted from $c a \mathrm{pH} 5.5$ for silica NPs $(x=1)$ to 3.5-4.0 for the estersilica samples. The main effect leading to more negative $\zeta$ potential values is the presence of a high density of carboxylic acid functions, up to $1.3 \mathrm{mmol} / \mathrm{g}$ for $x=0.8$. Indeed, the point of zero charge is shifted to a $\mathrm{pH}$ lower than the $\mathrm{pKa}$ of the carboxylic acid ( $c a 4$ 4.5). In particular, this confers them a very low $\zeta$ potential value at $\mathrm{pH} 10$ of $c a-40 \mathrm{mV}$ compared to $-30 \mathrm{mV}$ for pure silica NPs. A weaker effect affecting the $\zeta$ potential value is the electronic effect of the alkyl chain on silicon for silsesquioxanes, which lowers the acidity of the silanols. [20] This effect slightly counterbalances the carboxylic acid presence at low $\mathrm{pH}$, and explains why the pure silsesquioxane sample $(x=0)$ has a point of zero charge slightly higher than the silica/ester-silica mixtures.

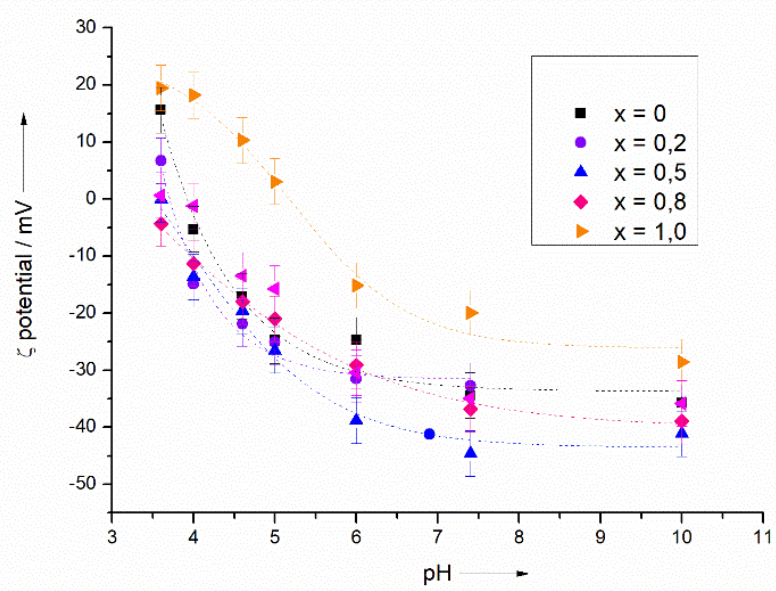

Figure 9: Influence of the $\mathrm{pH}$ on the $\zeta$ potential for NPs with $x=0$ (black squares); $x=0.2$ (red dots); $x=$ 0.5 (green triangles); $x=0.8$ (purple losanges) and $x=1$ (orange triangles). The measurements were performed in $2 \mathrm{mM}$ acetate or phosphate buffers, at a NP concentration of $0.1 \mathrm{mg} / \mathrm{mL}$.

Porous NPs with strongly negative $\zeta$ potential are indeed interesting to load cationic compounds.[34,36] Therefore, we decided to study the possible encapsulation of rhodamine B, a cationic dye, by these ester-silica NPs. Rhodamine B was incubated in pure water with concentrations of $1 \mathrm{mg} / \mathrm{mL}$ for both dye and NPs. Satisfactorily, the loading capacity of ester-silica NPs for rhodamine B (Table 3) was found to be always higher than that of pure silica NPs. In particular, the loading capacity is maximal for $x$ $=0.7$ with a value of $10.1 \mathrm{wt} \%$. This value was corroborated by TGA measurements (Figure S7), and the loading of rhodamine $\mathrm{B}$ within the pores was further suggested by $\mathrm{N}_{2}$-sorption measurements, with a strong decrease of both the specific surface area and the micropore volume (Figure S8). Two main factors are believed to affect the loading of this cationic dye: i) porosity and ii) electrostatic factors. While the specific surface area is similar for $x=0.7$ and $x=1$ (Table 2), these samples exhibit the extreme values in terms of loading capacity (Table 3), which confirms that the porosity is not the only factor influencing the loading capacity. Therefore, the presence of carboxylate functions (Table 3) is essential to promote a high loading capacity for cationic dyes, and a clear correlation can be drawn between those two factors (Figure S9). Indeed, the carboxylic acid content is very high for $x=0.7$, with $0.87 \mathrm{mmol} / \mathrm{g}$. Accordingly, the sample with $x=0.7$ exhibits the best balance between porosity and carboxylic acid loading. 

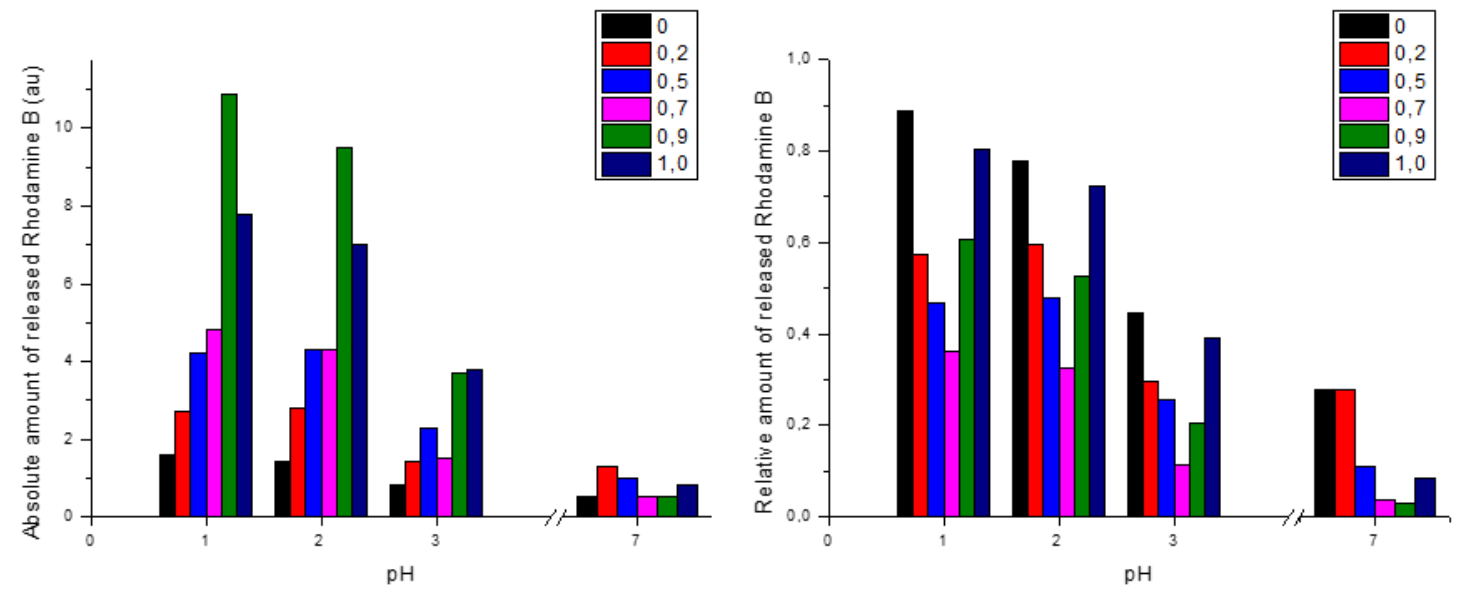

Figure 10: Variation of the absolute and relative amounts (compared to the encapsulated amount for all NPs) of rhodamine $\mathrm{B}$ released at various $\mathrm{pH}$ for the different molar fractions of silica, $x$.

The release of the organic cation in pure water was then probed (Figure 10). As expected, for the whole series of NPs, the amount released increases when the solution is acidified from pure water down to $\mathrm{pH} 2$, but then it remains constant until $\mathrm{pH} 1$. Furthermore, the dye remains strongly encapsulated within the NPs, only $37 \%$ being released at $\mathrm{pH} 1$ at equilibrium for the sample with $x=0.7$ (Figure 10b), despite this $\mathrm{pH}$ being much lower than the point of zero charge of the matrix. The relative amount of released rhodamine $\mathrm{B}$ is maximal for $x=0$, ie for pure non-porous bridged silsesquioxanes and $x=1$, ie pure silica. This corresponds to samples with the lowest amount of encapsulated dye. In the case of $x=0$, the dye can only be encapsulated at the surface by electrostatic and van der Waals forces owing to the lack of porosity. The relative liberated amount is minimal for $x=0.7$, a porous sample with the highest amount of carboxylic acid functions.

Thus, the elevated loading capacity for rhodamine B is directly related to the amount of carboxylic acid functions, which enable strong electrostatic forces between the cationic dye and the carboxylates. Moreover the loading capacity is favored by the strong organic character of NPs, which enhances the van der Waals forces, thereby preventing the release of the dye even at acidic $\mathrm{pH}$ where all carboxylate anions should be protonated.

\section{Conclusion:}

Monodisperse ester-silica nanoparticles with high amounts of organic groups were synthesized using a two-surfactant method at neutral $\mathrm{pH}$, which allows to limit the hydrolysis of the ester functions into carboxylic acid during the synthesis. In fact, most of this hydrolysis occurs during the moderately acidic treatment necessary to remove the surfactants. Interestingly, the size of these monodisperse NPs can be tuned depending on the hydrodynamic factors applied during the synthesis, such as stirring speed and stir bar size, which suggests that the nucleation of the very first clusters plays a key role in the NP synthesis. Interestingly, the fraction of organosilica precursor used during the synthesis has a significant influence on the textural properties of the obtained nanoparticles. As the silica fraction increases, mesopores are generated at the expense of the microporous volume. More specifically, for fractions lower than $x=0.7$, NPs show an absence of mesoporosity, which only appears for higher fractions, as evidenced by $\mathrm{N}_{2}$-sorption and visualized by TEM. These new materials constitute a rare example of porous organosilica nanoparticles obtained with a high content of organic groups. During the synthesis, but also during the washing steps, carboxylic acid functions are generated by ester hydrolysis, which can be monitored by FTIR. The high density of carboxylic acid functions, up to $1.4 \mathrm{mmol} / \mathrm{g}$, provides a very negative $\zeta$ potential to the NPs, even at neutral $\mathrm{pH}$. This surface chemistry property allows for an efficient encapsulation of rhodamine $\mathrm{B}$, a cationic dye for which a maximal loading capacity of $10 \%$ was measured for $x=0.7$, twice as high as for 
the pure silica sample. This efficient sorption capacity provides the prepared nanoparticles an interesting potential for drug delivery in nanomedicine application, or may be useful for the removal of cationic metals or organic pollutants from wastewater.

\section{Acknowledgements:}

This research did not receive any specific grant from funding agencies in the public, commercial, or not-for-profit sectors.

\section{References}

[1] A. Zamboulis, N. Moitra, J.J.E. Moreau, X. Cattoën, M. Wong Chi Man, Hybrid materials: versatile matrices for supporting homogeneous catalysts, J. Mater. Chem. 20 (2010) 9322-9338. https://doi.org/10.1039/c000334d.

[2] B. Lebeau, P. Innocenzi, Hybrid materials for optics and photonics, Chem. Soc. Rev. 40 (2011) 886-906. https://doi.org/10.1039/C0CS00106F.

[3] S. Parola, B. Julián-López, L.D. Carlos, C. Sanchez, Optical Properties of Hybrid OrganicInorganic Materials and their Applications, Adv. Funct. Mater. 26 (2016) 6506-6544. https://doi.org/10.1002/adfm.201602730.

[4] R.R. Castillo, D. Lozano, M. Vallet-Regí, Mesoporous Silica Nanoparticles as Carriers for Therapeutic Biomolecules, Pharmaceutics. 12 (2020). https://doi.org/10.3390/pharmaceutics12050432.

[5] P.N.E. Diagboya, E.D. Dikio, Silica-based mesoporous materials; emerging designer adsorbents for aqueous pollutants removal and water treatment, Microporous Mesoporous Mater. 266 (2018) 252-267. https://doi.org/https://doi.org/10.1016/j.micromeso.2018.03.008.

[6] F. Dong, L. Lu, C.-S. Ha, Silsesquioxane-Containing Hybrid Nanomaterials: Fascinating Platforms for Advanced Applications, Macromol. Chem. Phys. 220 (2019) 1800324. https://doi.org/10.1002/macp.201800324.

[7] P. Van Der Voort, D. Esquivel, E. De Canck, F. Goethals, I. Van Driessche, F.J. RomeroSalguero, Periodic Mesoporous Organosilicas: from simple to complex bridges; a comprehensive overview of functions $\{$,$\} morphologies and applications, Chem. Soc. Rev. 42$ (2013) 3913-3955. https://doi.org/10.1039/C2CS35222B.

[8] N. Mizoshita, T. Tani, S. Inagaki, Syntheses $\{$,$\} properties and applications of periodic mesoporous$ organosilicas prepared from bridged organosilane precursors, Chem. Soc. Rev. 40 (2011) 789-800. https://doi.org/10.1039/COCS00010H.

[9] M. Ferré, R. Pleixats, M. Wong Chi Man, X. Cattoën, Recyclable organocatalysts based on hybrid silicas, Green Chem. 18 (2016) 881-922. https://doi.org/10.1039/c5gc02579f.

[10] S. Shenoi-Perdoor, A. Noureddine, F. Dubois, M. Wong Chi Man, X. Cattoën, Click Functionalization of Sol-Gel Materials, in: L. Klein, M. Aparicio, A. Jitianu (Eds.), Handb. Sol-Gel Sci. Technol. Process. Charact. Appl., Springer International Publishing, Cham, 2018: pp. 30013040. https://doi.org/10.1007/978-3-319-32101-1_95.

[11] J. Alauzun, A. Mehdi, C. Reyé, R.J.P. Corriu, Mesoporous Materials with an Acidic Framework and Basic Pores. A Successful Cohabitation, J. Am. Chem. Soc. 128 (2006) 8718-8719. https://doi.org/10.1021/ja0622960.

[12] A. Noureddine, C.J. Brinker, Pendant/bridged/mesoporous silsesquioxane nanoparticles: Versatile and biocompatible platforms for smart delivery of therapeutics, Chem. Eng. J. 340 (2018) 125- 
147. https://doi.org/https://doi.org/10.1016/j.cej.2018.01.086.

[13] H. Li, M. Pérez-Trujillo, X. Cattoën, R. Pleixats, Recyclable Mesoporous Organosilica Nanoparticles Derived from Proline-Valinol Amides for Asymmetric Organocatalysis, ACS Sustain. Chem. Eng. 7 (2019) 14815-14828. https://doi.org/10.1021/acssuschemeng.9b02838.

[14] M. Gisbert-Garzarán, M. Vallet-Regí, Influence of the Surface Functionalization on the Fate and Performance of Mesoporous Silica Nanoparticles, Nanomaterials. 10 (2020). https://doi.org/10.3390/nano10050916.

[15] J.G. Croissant, X. Cattoën, J.-O. Durand, M. Wong Chi Man, N.M. Khashab, Organosilica hybrid nanomaterials with a high organic content: syntheses and applications of silsesquioxanes, Nanoscale. 8 (2016) 19945-19972. https://doi.org/10.1039/c6nr06862f.

[16] J.G. Croissant, X. Cattoën, M. Wong Chi Man, J.-O. Durand, N.M. Khashab, Syntheses and applications of periodic mesoporous organosilica nanoparticles, Nanoscale. 7 (2015) 20318-20334. https://doi.org/10.1039/c5nr05649g.

[17] C. Mauriello Jimenez, D. Aggad, J.G. Croissant, K. Tresfield, D. Laurencin, D. Berthomieu, N. Cubedo, M. Rossel, S. Alsaiari, D.H. Anjum, R. Sougrat, M.A. Roldan-Gutierrez, S. Richeter, E. Oliviero, L. Raehm, C. Charnay, X. Cattoën, S. Clément, M. Wong Chi Man, M. Maynadier, V. Chaleix, V. Sol, M. Garcia, M. Gary-Bobo, N.M. Khashab, N. Bettache, J.O. Durand, Porous Porphyrin-Based Organosilica Nanoparticles for NIR Two-Photon Photodynamic Therapy and Gene Delivery in Zebrafish, Adv. Funct. Mater. 28 (2018) 1800235. https://doi.org/10.1002/adfm.201800235.

[18] R. Bouchal, M. Daurat, M. Gary-Bobo, A. Da Silva, L. Lesaffre, D. Aggad, A. Godefroy, P. Dieudonné, C. Charnay, J.-O. Durand, P. Hesemann, Biocompatible Periodic Mesoporous Ionosilica Nanoparticles with Ammonium Walls: Application to Drug Delivery, ACS Appl. Mater. Interfaces. 9 (2017) 32018-32025. https://doi.org/10.1021/acsami.7b07264.

[19] J. Gehring, B. Trepka, N. Klinkenberg, H. Bronner, D. Schleheck, S. Polarz, Sunlight-Triggered Nanoparticle Synergy: Teamwork of Reactive Oxygen Species and Nitric Oxide Released from Mesoporous Organosilica with Advanced Antibacterial Activity, J. Am. Chem. Soc. 138 (2016) 3076-3084. https://doi.org/10.1021/jacs.5b12073.

[20] J. Croissant, X. Cattoën, M. Wong Chi Man, A. Gallud, L. Raehm, P. Trens, M. Maynadier, J.-O. Durand, Biodegradable Ethylene-Bis (Propyl) Disulfide-Based Periodic Mesoporous Organosilica Nanorods and Nanospheres for Efficient In-Vitro Drug Delivery, Adv. Mater. 26 (2014) 61746180. https://doi.org/https://doi.org/10.1002/adma.201401931.

[21] J.G. Croissant, Y. Fatieiev, N.M. Khashab, Degradability and Clearance of Silicon, Organosilica, Silsesquioxane, Silica Mixed Oxide, and Mesoporous Silica Nanoparticles, Adv. Mater. 29 (2017) 1604634. https://doi.org/10.1002/adma.201604634.

[22] L. Travaglini, P. Picchetti, R. Totovao, E.A. Prasetyanto, L. De Cola, Highly degradable iminedoped mesoporous silica particles, Mater. Chem. Front. 3 (2019) 111-119. https://doi.org/10.1039/C8QM00438B.

[23] Y. Fatieiev, J.G. Croissant, K. Julfakyan, L. Deng, D.H. Anjum, A. Gurinov, N.M. Khashab, Enzymatically degradable hybrid organic-inorganic bridged silsesquioxane nanoparticles for in vitro imaging, Nanoscale. 7 (2015) 15046-15050. https://doi.org/10.1039/C5NR03065J.

[24] J.G. Croissant, Y. Fatieiev, K. Julfakyan, J. Lu, A.-H. Emwas, D.H. Anjum, H. Omar, F. Tamanoi, J.I. Zink, N.M. Khashab, Biodegradable Oxamide-Phenylene-Based Mesoporous Organosilica Nanoparticles with Unprecedented Drug Payloads for Delivery in Cells, Chem. - A Eur. J. 22 (2016) 14806-14811. https://doi.org/10.1002/chem.201601714.

[25] L. Maggini, L. Travaglini, I. Cabrera, P. Castro-Hartmann, L. De Cola, Biodegradable PeptideSilica Nanodonuts, Chem. - A Eur. J. 22 (2016) 3697-3703.

https://doi.org/10.1002/chem.201504605. 
[26] J.L. Vivero-Escoto, W.J. Rieter, H. Lau, R.C. Huxford-Phillips, W. Lin, Biodegradable Polysilsesquioxane Nanoparticles as Efficient Contrast Agents for Magnetic Resonance Imaging, Small. 9 (2013) 3523-3531. https://doi.org/10.1002/smll.201300198.

[27] A. Corma, U. Díaz, M. Arrica, E. Fernández, Í. Ortega, Organic-Inorganic Nanospheres with Responsive Molecular Gates for Drug Storage and Release, Angew. Chemie Int. Ed. 48 (2009) 6247-6250. https://doi.org/10.1002/anie.200902208.

[28] H. Dong, L. Pang, H. Cong, Y. Shen, B. Yu, Application and design of esterase-responsive nanoparticles for cancer therapy, Drug Deliv. 26 (2019) 416-432. https://doi.org/10.1080/10717544.2019.1588424.

[29] K. Patel, S. Angelos, W.R. Dichtel, A. Coskun, Y.-W. Yang, J.I. Zink, J.F. Stoddart, EnzymeResponsive Snap-Top Covered Silica Nanocontainers, J. Am. Chem. Soc. 130 (2008) 2382-2383. https://doi.org/10.1021/ja0772086.

[30] N. Qiu, X. Liu, Y. Zhong, Z. Zhou, Y. Piao, L. Miao, Q. Zhang, J. Tang, L. Huang, Y. Shen, Esterase-Activated Charge-Reversal Polymer for Fibroblast-Exempt Cancer Gene Therapy, Adv. Mater. 28 (2016) 10613-10622. https://doi.org/10.1002/adma.201603095.

[31] L. Maggini, I. Cabrera, A. Ruiz-Carretero, E.A. Prasetyanto, E. Robinet, L. De Cola, Breakable mesoporous silica nanoparticles for targeted drug delivery, Nanoscale. 8 (2016) 7240-7247. https://doi.org/10.1039/C5NR09112H.

[32] R. Narayan, U.Y. Nayak, A.M. Raichur, S. Garg, Mesoporous Silica Nanoparticles: A Comprehensive Review on Synthesis and Recent Advances, Pharmaceutics. 10 (2018). https://doi.org/10.3390/pharmaceutics10030118.

[33] Q. He, X. Cui, F. Cui, L. Guo, J. Shi, Size-controlled synthesis of monodispersed mesoporous silica nano-spheres under a neutral condition, Microporous Mesoporous Mater. 117 (2009) 609_ 616. https://doi.org/https://doi.org/10.1016/j.micromeso.2008.08.004.

[34] C.-H. Tsai, W.-C. Chang, D. Saikia, C.-E. Wu, H.-M. Kao, Functionalization of cubic mesoporous silica SBA-16 with carboxylic acid via one-pot synthesis route for effective removal of cationic dyes, J. Hazard. Mater. 309 (2016) 236-248. https://doi.org/https://doi.org/10.1016/j.jhazmat.2015.08.051.

[35] A. Myerson, Handbook of Industrial Crystallization, Elsevier Science, 2002. https://books.google.fr/books?id=gJ7KNvbMtREC.

[36] D. Saikia, J.R. Deka, C.-E. Wu, Y.-C. Yang, H.-M. Kao, pH responsive selective protein adsorption by carboxylic acid functionalized large pore mesoporous silica nanoparticles SBA-1, Mater. Sci. Eng. C. 94 (2019) 344-356.

https://doi.org/https://doi.org/10.1016/j.msec.2018.09.043. 


\section{Graphical abstract}

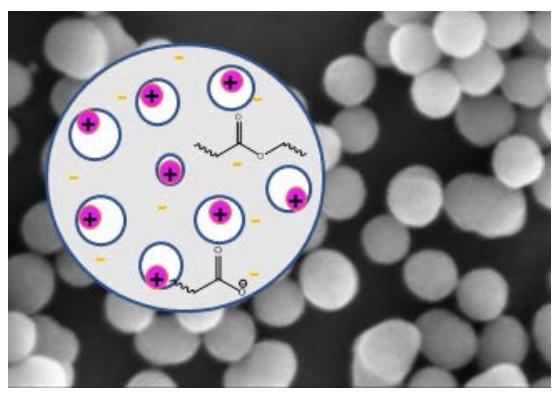

Porous ester-silica nanoparticles containing free carboxylic acid groups can be prepared and used for dye encapsulation.

Keywords: Nanoparticles, silicates, organosilica, ester, porous materials 